\title{
DINÂMICA DA DORMÊNCIA DE GEMAS DE CAQUIZEIRO ‘FUYU' EM REGIÃO DE BAIXA OCORRÊNCIA DE FRIO
}

\section{DORMANCY DYNAMICS OF 'FUYU' PERSIMMON TREE BUDS IN A REGION OF LOW CHILL OCCURRENCE}

\author{
Ruy Inacio Neiva de CARVALHO \\ Luiz Antonio BIASI ${ }^{2}$ \\ Flávio ZANETTE ${ }^{3}$ \\ José Carlos RENDOKE ${ }^{4}$ \\ Jean Magnus SANTOS ${ }^{5}$ \\ Gabriely Pinto PEREIRA ${ }^{5}$
}

\begin{abstract}
RESUMO
O objetivo da pesquisa foi determinar a dinâmica da dormência de gemas em caquizeiros cv. Fuyu durante o outono e inverno em região de baixa ocorrência de frio. Os ramos foram coletados em 10 datas no período de abril a agosto de 2007 e 2008 em um pomar do município de Fazenda Rio Grande, Paraná. Na última coleta, um grupo adicional de ramos foi coletado e mantido em refrigerador $\left(4\right.$ a $\left.7^{\circ} \mathrm{C}\right)$ por $672 \mathrm{~h}$. A intensidade de dormência foi avaliada pelo teste biológico de estacas de nós isolados realizado a temperatura de $25^{\circ} \mathrm{C}$ e fotoperíodo de $16 \mathrm{~h}$, por meio dos parâmetros tempo médio para brotação (TMB), taxa final de brotação (TF), tempo médio para aparecimento de folhas abertas (TMFA), taxa de brotações vigorosas (TBV) e velocidade de brotação (VB). O delineamento experimental adotado foi o completamente casualizado com 11 tratamentos, quatro repetições e dez estacas por parcela. Os dois anos de avaliação foram analisados individualmente. A endodormência mais intensa do caquizeiro 'Fuyu' ocorre no período compreendido entre a metade de maio e o início de junho em região de baixa ocorrência de frio.

Palavras-chave: Diospyros kaki; fisiologia vegetal; brotação; endodormência.
\end{abstract}

\section{ABSTRACT}

The objective of this work was to evaluate the dormancy dynamic of persimmon tree buds 'Fuyu' during autumn and winter in a region of low chill occurence. The stems were collected in an orchard in Fazenda Rio Grande, Parana State, Brazil, in 10 dates in the period from april to august of 2007 and 2008. In the last date, an addicional group of stems was collected and mainteined in a refrigerator $\left(4\right.$ a $\left.7^{\circ} \mathrm{C}\right)$ by $672 \mathrm{~h}$. The evaluation of dormancy was observed by the biological test of single node cuttings under controlled conditions $\left(25^{\circ} \mathrm{C}\right.$ and photoperiod of $16 \mathrm{~h}$ ) by the parameters average time for budburst (TMB), final rate of budburst (TF), average time for open leaves to appear (TMFA), rate of vigorous budburst (TBV) and velocity of budburst (VB). The entirely randomized experimental design was used with 11 treatments and four replications. The two years were analysed individually. The more intense endodormancy period of persimmon tree buds occure from mid may to early june in a region of low chill occurrence.

Key-words: Diospyros kaki; plant physiology; budburst; endodormancy.

1 Engenheiro Agrônomo, Dr., Bolsista de Produtividade em Pesquisa do CNPq, Professor Titular do Centro de Ciências Agrárias e Ambientais da Pontifícia Universidade Católica do Paraná, Campus São José dos Pinhais. Rodovia BR 376, km 14, CEP 83010-500, São José dos Pinhais, Paraná, Brasil. E-mail: ruy.carvalho@pucpr.br. Autor para correspondência.

2 Engenheiro Agrônomo, Dr., Professor Associado do Departamento de Fitotecnia e Fitossanitarismo, Universidade Federal do Paraná (UFPR). Bolsista de Produtividade em Pesquisa do CNPq; Curitiba, Paraná, Brasil. E-mail: biasi@ufpr.br

${ }^{3}$ Engenheiro Agrônomo, Dr., Professor do Departamento de Fitotecnia e Fitossanitarismo da Universidade Federal do Paraná (UFPR), Bolsista de Produtividade em Pesquisa do CNPq; Curitiba, Paraná, Brasil. E-mail: flazan@ufpr.br

${ }^{4}$ Engenheiro Agrônomo, autônomo, São José dos Pinhais, Paraná, Brasil. E-mail: jcrendoke@yahoo.com.br

${ }^{5}$ Estudante de agronomia da Pontifícia Universidade Católica do Paraná (PUC-PR), São José dos Pinhais, Paraná, Brasil. Bolsista PIBIC. Email: jmferoldi@yahoo.com.br; gabyp.pereira@hotmail.com 
CARVALHO, R.I.N. et al. Dinâmica da dormência de gemas de caquizeiro...

\section{INTRODUÇÃO}

A dormência de gemas estende-se ao longo do ano passando pelas fases de ecodormência, na qual um fator ambiental desfavorável limita o crescimento, de paradormência, em que a inibição de crescimento é causada pela influência de outros órgãos da planta, e de endodormência, quando eventos bioquímicos e fisiológicos a níveis meristemáticos induzem a ausência de brotação (Lang et al., 1987).

Nos pomares, a endodormência é a fase mais importante, pois se não for superada adequadamente resulta em brotação heterogênea e limitações do crescimento vegetativo e frutificação da planta. Esta fase pode ser também influenciada por alterações morfológicas e ambientais, uma vez que a gema é um broto embrionário constituído por um meristema apical, nós internos e folhas rudimentares envolvidos por escamas para prevenção ao dessecamento, restrição ao movimento do oxigênio e manutenção de calor (Raven et al., 2001).

Diversas espécies frutíferas de clima temperado vêm sendo estudadas para conhecimento dos aspectos envolvidos na entrada, manutenção e liberação da endodormência como a macieira (Carvalho \& Zanette, 2004b; Putti et al., 2003), a pereira (Oliveira et al., 2008) e o pessegueiro (Oliveira Filho \& Carvalho, 2003). O caquizeiro é uma cultura pouco estudada em relação à dormência e os resultados não convergem para um entendimento claro da sua fisiologia, pois enquanto Mowat (1995) concluiu que gemas de caquizeiro podem sair da dormência mesmo sem ocorrência de frio, Faquim et al. (2007) evidenciaram que as gemas da caquizeiro necessitam de $504 \mathrm{~h}$ de frio para atingir $100 \%$ de brotação de gemas terminais e laterais.

As diversas teorias a respeito da instalação e liberação da endodormência postulam que este evento fisiológico pode estar relacionado com alterações do metabolismo energético na gema (Bonhomme et al., 2000), conteúdo de proteínas (Tamura et al., 1998), fluxo de carboidratos, suprimento de nutrientes e translocação de reservas a curta distância (Carvalho \& Zanette, 2004a; Marquat et al., 1999), regulação hormonal (Stafstrom, 2000), bem como com uma relação entre todos estes fatores. As temperaturas ocorridas no ambiente podem afetar cada um destes eventos distintamente, de forma a dificultar a avaliação do efeito específico de uma determinada temperatura na dinâmica da dormência.

As diferentes regiões paranaenses indicadas como aptas para o cultivo de fruteiras de clima temperado são bastante distintas em termos de quantidade de frio acumulada. A região dos municípios de Ponta Grossa e Curitiba, onde se encontra o município de Fazenda Rio Grande, é considerada de baixa ocorrência de frio e ocorre em média de 161,8 a $169,2 \mathrm{~h}$ de frio abaixo de $7,2{ }^{\circ} \mathrm{C}$ (Botelho et al., 2006). Segundo Petri et al. (1996) a exigência em frio da maior parte das cultivares de fruteiras de clima temperado é superior a $300 \mathrm{~h}$.

Diversas formas de quantificação de frio ocorrido em uma região foram desenvolvidas para avaliar a aptidão da região ao cultivo de frutíferas gerando zoneamentos climáticos para algumas espécies, como o pessegueiro no Paraná (Caramori et al., 2008). Dentre as formas de estimativa de frio destacam-se o acúmulo de horas de frio abaixo de $7,2{ }^{\circ} \mathrm{C}$ e o acúmulo de unidades de frio calculadas com base em temperaturas horárias ocorridas durante o outono e inverno, gerando os modelos Utah e Carolina do Norte, os quais sofreram alterações para adequação a regiões com inverno mais ameno (Petri et al., 1996; Richardson et al., 1974; Shaltout \& Unrath, 1983). O conhecimento da relação entre as temperaturas ocorridas e a intensidade da dormência das gemas é essencial para se estimar a adaptação da espécie frutífera, bem como sua fisiologia de entrada e saída da endodormência.

O objetivo da pesquisa foi determinar a dinâmica da dormência de gemas de caquizeiro cv. Fuyu em região de baixa ocorrência de frio.

\section{MATERIAL E MÉTODOS}

O experimento foi realizado nos anos de 2007 e 2008, entre os meses de abril a agosto. Ramos mistos de um ano, íntegros, sadios e de posição oblíqua na periferia da copa foram coletados de plantas de 14 anos de idade, com crescimento homogêneo, no pomar de caquizeiro cv. Fuyu enxertado sobre Diospyros virginiana, da Fazenda Experimental Gralha Azul da Pontifícia Universidade Católica do Paraná (PUCPR), no município de Fazenda Rio Grande, Paraná (latitude de $25^{\circ} 39^{\prime}$ Sul, longitude de $49^{\circ} 16^{\prime}$ 'Oeste e altitude média de $895 \mathrm{~m}$ ). Para avaliação do frio natural ocorrido foram calculados o número de horas de frio $\left(\leq 7,2^{\circ} \mathrm{C}\right)$ e as unidades de frio ocorridas de acordo com o modelo de Utah e Utah modificado (Richardson et al., 1974) e com uso do programa computacional "Hora Frio", do Departamento de Agrometeorologia da Empresa Catarinense de Pesquisa Agropecuária (EPAGRI) com base nos dados meteorológicos fornecidos pelo SIMEPAR (2008).

Foram realizadas coletas de 40 ramos a cada duas semanas a partir do dia 04 de abril de 2007 e 07 de abril de 2008 até o início de brotação das plantas ocorrido em agosto, totalizando dez coletas. Na última coleta, um grupo adicional de ramos foi coletado e mantido em refrigerador a temperatura de 4 a $7{ }^{\circ} \mathrm{C}$ por $672 \mathrm{~h}$, para fornecimento de frio necessário para liberação natural da dormência.

A avaliação da dormência foi realizada por meio do teste biológico em câmaras de crescimento (BOD) a temperatura de $25^{\circ} \mathrm{C}$ e fotoperíodo de 16 h (Faquim et al., 2007). A porção mediana dos ramos foi dividida em estacas de $7 \mathrm{~cm}$ de comprimento, mantendo-se nas mesmas apenas uma gema na parte superior da estaca. Nas coletas de abril a maio, as folhas presentes foram retiradas 
com tesoura e o fragmento do pecíolo mantido unido à estaca para queda natural ao longo das observações. Não foram coletados ramos com frutos ou com cicatrizes de frutos. Cada parcela experimental foi formada por dez estacas mantidas em vasos plásticos com vermiculita umedecida como substrato. Os vasos foram cobertos com filme plástico de PVC com cinco orifícios de $2 \mathrm{~mm}$ para ventilação. Os testes foram realizados com quatro repetições, totalizando 40 estacas por data de avaliação.

As estacas foram avaliadas individualmente a cada dois dias, até um período máximo de 40 dias, de acordo com os estádios de crescimento da gema vegetativa "Ponta Verde" (PV) (aparecimento de modificações na coloração da gema, ficando esta com o ápice amareloesverdeado) e "Gema Aberta" (GAb) (aparecimento de folhas abertas). Com base nestes estádios, foram calculados o tempo médio para brotação (TMB) que representa o número médio de dias passados entre a instalação do experimento e a detecção do estádio PV, a taxa final de brotação (TF) que representa a porcentagem de estacas com gemas que atingiram PV, a taxa de brotações vigorosas (TBV), que representa a porcentagem de estacas com gemas que apresentaram o estádio PV e evoluíram até o estádio Gab [TBV $=(\%$ de estacas com gemas no estágios Gab) x 100/TF] e a velocidade de brotação (VB) que avalia a brotação das gemas em função do tempo para a brotação de acordo com a equação $V B=\Sigma\left(n_{i} / t_{i}\right)$ (gemas/dia) em que $\mathrm{n}_{\mathrm{i}}=$ número de gemas que atingiram o estádio PV no tempo "i", e $\mathrm{t}_{\mathrm{i}}=$ tempo após instalação do teste $(\mathrm{i}=1 \rightarrow 40)$.

O delineamento experimental adotado foi o completamente casualizado com 11 tratamentos e quatro repetições. Os dois anos de avaliação foram analisados individualmente. As médias dos tratamentos, com diferença significativa pelo teste $\mathrm{F}$ na análise de variância, foram submetidas ao teste Tukey ao nível de significância de 5\%.

\section{RESULTADOS E DISCUSSÃO}

Nas três primeiras coletas, de abril a início de maio, não houve brotações de gemas no teste biológico realizado em 2007 e apenas brotações esporádicas de até $5 \%$ das gemas após 40 dias de observação em 2008 (Tabela 1). Segundo Faquim et al. (2007), gemas laterais de caquizeiro coletadas em abril apresentaram apenas brotações esporádicas de $3,3 \%$ durante o teste biológico mesmo após o fornecimento de até $672 \mathrm{HF}$. A ausência de brotação em condições ótimas para crescimento evidencia uma fase da dormência. A paradormência refere-se à influência de outro órgão do vegetal sobre a gema (Lang et al., 1987) e nas primeiras datas de coleta as plantas continham folhas e frutos. Embora não tenham sido coletados ramos com frutos e no preparo das estacas para o teste as folhas tenham sido cortadas, permanecendo apenas um fragmento de pecíolo ligado à base da gema, estas ações podem não ter impedido o efeito inibitório já manifestado em toda a planta por influência de folhas e frutos presentes. Em condições naturais, esta inibição só seria superada pela colheita ou queda natural das folhas que consiste em alterações hormonais internas para formação da zona de abscisão foliar. Assim, a paradormência do caquizeiro é intensa e de difícil superação. No campo, a colheita e queda de folhas podem marcar o fim da paradormência e o início da endodormência do caquizeiro.

A distribuição de fotoassimilados no caquizeiro para outros drenos em detrimento às gemas, como considerado por Carvalho \& Alves (2007), pode ser uma importante causa que, juntamente com a redução do fotoperíodo, induz as gemas à dormência. Com a colheita do fruto, esta relação é eliminada, porém a dormência já instalada pode passar a sofrer influência da translocação de reservas a distâncias mais próximas, como porções de ramos adjacentes às gemas.

A endodormência mais intensa foi caracterizada pelo elevado TMB (34,3 dias) na segunda quinzena de maio de 2007 (Tabela 1), época em que as plantas não continham mais frutos e folhas. Em 2008, o TMB mais elevado foi de 31 dias e se manteve alto até início de junho. Estes resultados estão de acordo com os obtidos por Carvalho \& Alves (2007), que encontraram TMB de 31 dias na dormência mais intensa de gemas de caquizeiro em abril e maio, e por Faquim et al. (2007), que encontraram TMB máximo de 34,8 dias ao final de maio, ambos com a cultivar Fuyu em condições de baixa ocorrência de frio. Nos dois anos estudados, na endodormência mais intensa, a TF e a VB foram baixas, evidenciando a baixa aptidão da gema ao crescimento. Na saída da endodormência, a TF foi superior a $95 \%$ e a VB superior a 0,69 gemas $\mathrm{dia}^{-1}$, valores que podem ser considerados elevados por estarem associados à ausência de endodormência (Tabela 1).

Considera-se que a obtenção de TMB abaixo de 14 dias seja indicativa de ausência de endodormência, em especial se aliada a TF acima de $90 \%$. Carvalho \& Alves (2007) consideraram a transição da endodormência para a ecodormência de gemas de caquizeiro quando o TMB foi reduzido para próximo de 20 dias e Faquim et al. (2007) encontraram TMB mínimo de 17,2 dias em agosto em região de baixa ocorrência de frio. $O$ estudo do TMB isoladamente pode levar a imprecisões de interpretação uma vez que, embora apresente valores elevados, quando associado a baixas TF e VB é indicativo de inaptidão à brotação homogênea de gemas.

Na saída natural da endodormência, sem o tratamento com frio adicional, a TF foi elevada, acima de $80 \%$, e a VB foi acima de 0,69 gemas dia $^{-1}$. A TBV não se caracterizou como um parâmetro complementar adequado para avaliação da endodormência uma vez que em 2007 foi elevada em todo o período analisado e o tratamento com frio adicional prejudicou o crescimento das brotações novas. A variação da TBV em 2008 foi desuniforme, sem associação definida com os 
CARVALHO, R.I.N. et al. Dinâmica da dormência de gemas de caquizeiro...

TABELA 1 - Tempo médio para brotação (TMB), taxa final de brotação (TF), velocidade de brotação (VB) e taxa de brotações vigorosas (TBV) em estacas de nós isolados de caquizeiro 'Fuyu' coletadas em Fazenda Rio Grande, Paraná, 2007 e 2008.

\begin{tabular}{|c|c|c|c|c|c|}
\hline $\begin{array}{c}\text { Datas de } \\
\text { coleta (2007) }\end{array}$ & $\begin{array}{r}\mathrm{TN} \\
\text { (dia }\end{array}$ & & $\begin{array}{c}\text { TF } \\
(\%)^{\star *}\end{array}$ & $\begin{array}{c}\text { VB } \\
\left(\text { gemas dia }{ }^{-1}\right)^{\star \star}\end{array}$ & $\begin{array}{l}\text { TBV } \\
(\%)^{* *}\end{array}$ \\
\hline $04 / 04$ & - & $* * *$ & 0,0 & 0 & - \\
\hline $18 / 04$ & - & & 0,0 & 0 & - \\
\hline $02 / 05$ & - & & 0,0 & 0 & - \\
\hline $16 / 05$ & 34,3 & a & $25,0 \quad b$ & 0,08 & $81,3 a b$ \\
\hline $30 / 05$ & 31,4 & a & $40,0 \quad b$ & 0,13 & 100,0 a \\
\hline $13 / 06$ & 25,8 & $\mathrm{~b}$ & 85,0 a & $0,34 \quad b$ & 100,0 a \\
\hline $27 / 06$ & 25,2 & $b$ & $27,5 \quad b$ & 0,11 & 100,0 a \\
\hline $11 / 07$ & 22,9 & $\mathrm{bc}$ & 80,0 a & $0,36 \quad b$ & 100,0 a \\
\hline $25 / 07$ & 19,2 & c & 90,0 a & $0,42 \quad b$ & 100,0 a \\
\hline 08/08 & 13,4 & $d$ & 100,0 a & 0,74 a & 100,0 a \\
\hline 08/08+Frio* & 12,2 & d & 100,0 a & $0,42 \quad b$ & $50,0 \quad b$ \\
\hline CV (\%) & 7,28 & & 19,95 & 22,07 & 16,29 \\
\hline \multicolumn{6}{|l|}{$(2008)$} \\
\hline $07 / 04$ & 35,0 & $* * *$ & 2,5 & 0,01 & 0,0 \\
\hline $23 / 04$ & - & & 0,0 & 0 & - \\
\hline $05 / 05$ & 30,0 & & 5,0 & 0,02 & 0,0 \\
\hline $19 / 05$ & 31,0 & a & $52,5 \quad c$ & 0,17 & $44,3 \quad b c$ \\
\hline $02 / 06$ & 25,9 & $a b$ & $60,0 \quad b c$ & 0,23 & 56,0 abc \\
\hline $16 / 06$ & 19,6 & $\mathrm{bc}$ & 92,5 a & 0,49 bcd & $84,4 \quad a b$ \\
\hline $30 / 06$ & 19,5 & bc & $85,0 a b$ & $0,47 \quad \mathrm{~cd}$ & 54,4 bc \\
\hline $14 / 07$ & 14,6 & c & $100,0 a$ & $0,69 a b c$ & 97,5 a \\
\hline $28 / 07$ & 15,1 & c & 97,5 a & $0,69 a b c$ & 43,3 \\
\hline $08 / 08$ & 16,4 & c & 95,0 a & $0,83 a b$ & 15,0 \\
\hline 08/08+Frio* & 13,8 & c & 92,5 a & 0,99 a & $58,0 \quad a b$ \\
\hline CV (\%) & 16,19 & & 13,47 & 25,70 & 31,61 \\
\hline
\end{tabular}

${ }^{*}$ Tratamento com $672 \mathrm{~h}$ de frio de 4 a $7{ }^{\circ} \mathrm{C}$.

**Médias seguidas por letras distintas nas colunas diferem entre si pelo Teste Tukey ao nível de significância de $5 \%$.

${ }^{* * *}$ As três primeiras datas não foram consideradas na análise estatística em função de haver nenhuma ou apenas brotações esporádicas até 40 dias.

Valores de DMS a $5 \%$

Variável TMB (2007): 3,93 Variável TMB (2008): 7,39

Variável TF (2007): 31,95 Variável TF (2008): 26,59

Variavel VB (2007): $0,17 \quad$ Variavel VB (2008): 0,34

Variável TBV (2007): 34,83 Variável TBV (2008): 41,88

outros parâmetros (Tabela 1).

A época de dormência mais intensa varia entre as espécies frutíferas de clima temperado em regiões de baixa ocorrência de frio, pois enquanto gemas de pessegueiro, assim como as de caquizeiro, apresentaram dormência mais intensa no começo de maio (Oliveira Filho \& Carvalho, 2003), gemas de macieira tiveram dormência mais intensa em julho (Carvalho \& Zanette, 2004b).

Por qualquer forma de avaliação de frio ocorrido na região, até a metade de maio ocorreram apenas 6 HF em 2007 e 3 HF em 2008 (Tabela 2). A avaliação horária de todas as temperaturas ocorridas e sua transformação em UF pelos métodos Utah e Utah modificado, que atribui um fraco efeito anulador de frio às altas temperaturas, resultou em UF negativas (Tabela 3). Mesmo com o uso do programa Hora Frio que antes do período de frio não considera efeito anulador de frio pelas altas temperaturas, foram registradas 0,5 UF em 2007 e 0 UF em 2008 até metade de maio (Tabela 4). Estes fatores indicam que a transição da paradormência para a endodormência independe das baixas temperaturas e deve ser influenciada por outro fator ambiental, como a redução do fotoperíodo nesta época do ano. Carvalho \& Alves (2007) também indicaram a dormência mais intensa do caquizeiro quando havia ocorrido zero HF e UF negativas. 
CARVALHO, R.I.N. et al. Dinâmica da dormência de gemas de caquizeiro...

TABELA 2 - Horas de frio abaixo de $7,2^{\circ} \mathrm{C}$ em pomar de caquizeiro 'Fuyu' em diferentes datas em Fazenda Rio Grande, Paraná, 2007 e 2008.

\begin{tabular}{|c|c|c|c|c|c|}
\hline \multirow{2}{*}{$\begin{array}{l}\text { Períodos } \\
2007\end{array}$} & \multicolumn{2}{|c|}{ Horas de frio $\leq 7,2^{\circ} \mathrm{C}^{*}$} & \multirow{2}{*}{$\begin{array}{c}\text { Períodos } \\
2008\end{array}$} & \multicolumn{2}{|c|}{ Horas de frio $\leq 7,2^{\circ} \mathrm{C}^{*}$} \\
\hline & Entre as datas & Acumuladas & & $\begin{array}{c}\text { Entre as } \\
\text { datas }\end{array}$ & Acumuladas \\
\hline $01 / 04$ a 04/04 & 0 & 0 & $01 / 04$ a $07 / 04$ & 0 & 0 \\
\hline 04/04 a 18/04 & 0 & 0 & 07/04 a 23/04 & 0 & 0 \\
\hline $18 / 04$ a $02 / 05$ & 0 & 0 & $23 / 04$ a $05 / 05$ & 0 & 0 \\
\hline 02/05 a 16/05 & 6 & 6 & 05/05 a 19/05 & 3 & 3 \\
\hline $16 / 05$ a $30 / 05$ & 17 & 23 & $19 / 05$ a $02 / 06$ & 1 & 4 \\
\hline $30 / 05$ a $13 / 06$ & 41 & 64 & $02 / 06$ a $16 / 06$ & 20 & 24 \\
\hline $13 / 06$ a $27 / 06$ & 0 & 64 & $16 / 06$ a $30 / 06$ & 40 & 64 \\
\hline $27 / 06$ a $11 / 07$ & 5 & 69 & $30 / 06$ a $14 / 07$ & 0 & 64 \\
\hline $11 / 07$ a $25 / 07$ & 27 & 96 & $14 / 07$ a $28 / 07$ & 12 & 76 \\
\hline 25/07 a 08/08 & 65 & 161 & $28 / 07$ a $08 / 08$ & 0 & 76 \\
\hline
\end{tabular}

${ }^{*}$ Cálculos efetuados com base em dados fornecidos pelo SIMEPAR $(2007,2008)$.

TABELA 3 - Unidades de frio (UF) calculadas pelos modelos Utah e Utah modificado ocorridas no pomar de caquizeiro 'Fuyu' em diferentes datas de coleta em Fazenda Rio Grande, Paraná, 2007 e 2008.

\begin{tabular}{|c|c|c|c|c|}
\hline \multirow{2}{*}{$\begin{array}{c}\text { Períodos } \\
(2007)\end{array}$} & \multicolumn{2}{|c|}{ UF (Modelo Utah) } & \multicolumn{2}{|c|}{ UF (Modelo Utah modificado) } \\
\hline & Entre as datas & Acumuladas & Entre as datas & Acumuladas \\
\hline $01 / 04$ a 04/04 & $-84,5$ & $-84,5$ & $-50,0$ & $-50,0$ \\
\hline 04/04 a 18/04 & $-247,0$ & $-331,5$ & $-67,0$ & $-117,0$ \\
\hline $18 / 04$ a $02 / 05$ & $-243,5$ & $-575,0$ & $-74,5$ & $-191,5$ \\
\hline 02/05 a 16/05 & $-111,5$ & $-686,5$ & $-5,0$ & $-196,5$ \\
\hline $16 / 05$ a $30 / 05$ & $-29,5$ & $-716,0$ & 25,5 & $-171,0$ \\
\hline $30 / 05$ a $13 / 06$ & $-20,0$ & $-736,0$ & 47,0 & $-124,0$ \\
\hline $13 / 06$ a $27 / 06$ & -116 & $-852,0$ & $-40,5$ & $-164,5$ \\
\hline $27 / 06$ a $11 / 07$ & $-82,5$ & $-934,5$ & $-4,0$ & $-168,5$ \\
\hline $11 / 07$ a $25 / 07$ & 26,5 & $-908,0$ & 69,5 & $-99,0$ \\
\hline $25 / 07$ a $08 / 08$ & 103,5 & $-804,5$ & 124,0 & 25,0 \\
\hline \multicolumn{5}{|l|}{$(2008)$} \\
\hline $01 / 04$ a $07 / 04$ & $-80,5$ & $-80,5$ & $-25,0$ & $-25,0$ \\
\hline 07/04 a 23/04 & $-210,0$ & $-290,5$ & $-63,5$ & $-88,5$ \\
\hline $23 / 04$ a $05 / 05$ & $-81,5$ & $-372,0$ & $-20,5$ & $-109,0$ \\
\hline 05/05 a 19/05 & 26,0 & $-346,0$ & 76,0 & $-33,0$ \\
\hline $19 / 05$ a $02 / 06$ & $-107,0$ & $-453,0$ & $-27,5$ & $-60,5$ \\
\hline 02/06 a 16/06 & $-59,5$ & $-512,5$ & 7,5 & $-53,0$ \\
\hline $16 / 06$ a $30 / 06$ & 35,0 & $-477,5$ & 73,5 & 20,5 \\
\hline $30 / 06$ a $14 / 07$ & $-46,0$ & $-523,5$ & 22,0 & 42,5 \\
\hline $14 / 07$ a $28 / 07$ & $-57,5$ & $-581,0$ & 9,5 & 52,0 \\
\hline 28/07 a 08/08 & $-50,5$ & $-631,5$ & 0,5 & 52,5 \\
\hline
\end{tabular}


CARVALHO, R.I.N. et al. Dinâmica da dormência de gemas de caquizeiro...

TABELA 4 - Unidades de frio (UF), calculadas pelo programa Hora Frio com base no modelo Utah modificado, ocorridas no pomar de caquizeiro 'Fuyu' em diferentes datas em Fazenda Rio Grande, Paraná, 2007 e 2008.

\begin{tabular}{cccc}
\hline $\begin{array}{c}\text { Datas de coleta } \\
(2007)\end{array}$ & $\begin{array}{c}\text { UF acumuladas (Modelo } \\
\text { Utah modificado) }\end{array}$ & $\begin{array}{c}\text { Datas de coleta } \\
(2008)\end{array}$ & $\begin{array}{c}\text { UF acumuladas (Modelo } \\
\text { Utah modificado) }\end{array}$ \\
\hline $04 / 04$ & 0,0 & $07 / 04$ & 0,0 \\
$18 / 04$ & 0,0 & $23 / 04$ & 0,0 \\
$02 / 05$ & 0,0 & $05 / 05$ & 9,5 \\
$16 / 05$ & 0,5 & $19 / 05$ & 0,0 \\
$30 / 05$ & 64,5 & $02 / 06$ & 41,0 \\
$13 / 06$ & 69,5 & $16 / 06$ & 24,5 \\
$27 / 06$ & 64,5 & $30 / 06$ & 48,5 \\
$11 / 07$ & 64,0 & $14 / 07$ & 0,0 \\
$25 / 07$ & 121,0 & $28 / 07$ & 0,0 \\
$08 / 08$ & 207,5 & $08 / 08$ & 5,5 \\
\hline
\end{tabular}

Em 2007, a redução do TMB foi gradual de junho até o início de agosto, enquanto em 2008 a redução do TMB foi mais abrupta, obtendo-se na metade de junho valores semelhantes aos obtidos em agosto (Tabela 1). Nos dois anos, o tratamento das estacas com frio adicional não alterou o TMB, indicando que as condições ambientais ocorridas na região propiciaram a superação natural da endodormência das gemas. Segundo Faquim et al. (2007), as gemas de caquizeiro 'Fuyu' apresentaram $100 \%$ de brotação de gemas laterais e terminais quando receberam $504 \mathrm{~h}$ de frio de 4 a $7{ }^{\circ} \mathrm{C}$ em geladeira. Na região de coleta de ramos, ocorreram em 2007 e 2008 apenas 161 HF e 76 HF (Tabela 2), respectivamente, e a brotação ocorreu naturalmente. Esta divergência pode indicar que quando se mantém todos os fatores ambientais controlados e invariáveis, a baixa temperatura passa a ter importância maior. Em condições de campo, as alterações ambientais, em especial a duração e intensidade da luz incidente sobre o pomar, induzem menor requerimento em frio. Plantas expostas a condições de dias longos podem apresentar maior número de gemas abertas, pois a luz possivelmente atua no decréscimo do nível de inibidores de crescimento (Petri et al., 1996).

Os modelos de cálculos de UF indicaram baixa ocorrência de frio na região aliada à ocorrência de períodos com temperaturas elevadas. No modelo de Utah, temperaturas acima de $16{ }^{\circ} \mathrm{C}$ já possuem efeito anulador de frio e no modelo de Utah modificado, que minimiza o efeito negativo de altas temperaturas em regiões subtropicais, temperaturas acima de $21{ }^{\circ} \mathrm{C}$ tem efeito anulador de frio. Mesmo com eliminação do efeito prolongado do calor como anulador de frio considerado pelo programa Hora Frio, as unidades de frio acumuladas foram de apenas 207,5 UF em 2007 e de 5,5 UF em 2008 (Tabela 4).

\section{CONCLUSÃO}

A endodormência mais intensa do caquizeiro 'Fuyu' ocorre no período da metade de maio ao início de junho em região de baixa ocorrência de frio.

\section{REFERÊNCIAS}

1. BONHOMME, M.; RAGEAU, R.; GENDRAUD, M. ATP, ADP and NTP contents in vegetative and floral peach buds during winter: are they useful for characterizing the type of dormancy? In: VIÉMONT, J.-D.; CRABBÉ, J. (Ed.). Dormancy in plants: from whole plant behaviour to cellular control. Cambridge: University Press, 2000. p. 245-257.

2. BOTELHO, R. V.; AYUB, R. A.; MÜLLER, M. M. L. Somatória de horas de frio e de unidades de frio em diferentes regiões do estado do Paraná. Scientia Agraria, v. 7, n. 1-2, p. 89-96, 2006.

3. CARAMORI, P. H. et al. Zoneamento agroclimático para o pessegueiro e a nectarineira no Estado do Paraná. Revista Brasileira de Fruticultura, v. 30, n. 4, p. 1040-1044, 2008.

4. CARVALHO, R. I. N.; ALVES, M. C. A. Intensidade de dormência das gemas de caquizeiro 'Fuyu' no período do outono e inverno na região de Fazenda Rio Grande - PR. Revista Brasileira de Agrociência, v. 13, n.1, p. 35-38, 2007.

5. CARVALHO, R. I. N.; ZANETTE, F. Conteúdo de carboidratos em gemas e ramos de macieira durante o outono e inverno em região de baixa ocorrência de frio. Revista Brasileira de Fruticultura, v. 26, n. 2, p. 202-205, $2004 a$.

6. CARVALHO, R. I. N.; ZANETTE, F. Dinâmica da dormência de gemas de macieira 'Imperial Gala' durante o outono e inverno em região de baixa ocorrência de frio. Revista Brasileira de Fruticultura, v. 26, n. 1, p. 65-68, 2004b.

7. FAQUIM, R.; SILVA, I. D.; CARVALHO, R. I. N. Necessidade de frio para quebra de dormência de gemas de caquizeiro 'Fuyu'. Revista Brasileira de Fruticultura, v. 29, n.3, p. 438-444, 2007. 
CARVALHO, R.I.N. et al. Dinâmica da dormência de gemas de caquizeiro...

8. LANG, G. A. et al. Endo-, para- and ecodormancy: physiological terminology and classification for dormancy research Hortscience, v. 22, n. 3, p. 371-378, 1987.

9. MARQUAT, C. et al. Dormancy in vegetative buds of peach: relation between carbohydrate absorption potentials and carbohydrate concentration in the bud during dormancy and its release. Scientia Horticulturae, v. 79, n. 3-4, p. 151-162, 1999.

10. MOWAT, A. D. The effect of root temperature on bud dormancy release of persimmon (Diospyros kaki L.). Acta Horticulturae, v. 1, n. 409, p. 137-140, 1995.

11. OLIVEIRA FILHO, P. R. C.; CARVALHO, R. I. N. Dinâmica da dormência em gemas de pessegueiro das variedades Eldorado e Ágata. Revista Acadêmica, v. 1, n. 3, p. 41-46, 2003.

12. OLIVEIRA, O. R. et al. Quebra de dormência de pereira 'Hosui' com uso de óleo mineral em dois tipos de condução. Revista Brasileira de Fruticultura, v. 30, n. 2, p. 409-413, 2008.

13. PETRI, J. L. et al. Dormência e indução da brotação de fruteiras de clima temperado. Florianópolis: Epagri, 1996. 110 p. (Boletim Técnico, 75).

14. PUTTI, G. L.; PETRI, J. L.; MENDEZ, M. E. Temperaturas efetivas para a dormência da macieira (Malus domestica Borkh.). Revista Brasileira de Fruticultura, v. 25, n. 2, p. 210-212, 2003.

15. RAVEN, P. H.; EVERT, R. F.; EICHHORN, S. E. Biologia vegetal. 6. ed. Rio de Janeiro: Guanabara Koogan, 2001. 906

16. RICHARDSON, E. A.; SEELEY, S. D.; WALKER, D. R. A model for estimating the completion of rest for 'Redhaven' and 'Elbert' peach trees. HortScience, v. 9, n. 4, p. 331-332, 1974.

17. SHALTOUT, A. D.; UNRATH, C. R. Rest completion prediction model for 'Starkrimson Delicious' apples. Journal of the American Society for Horticultural Science, v. 108, n. 6, p. 957-961, 1983.

18. SIMEPAR Instituto Tecnológico SIMEPAR. Dados meteorológicos. Curitiba: SIMEPAR, 2007. 1 CD.

19. SIMEPAR Instituto Tecnológico. Dados meterológicos. Curitiba: SIMEPAR, 2008. 1 CD.

20. STAFSTROM, J. P. Regulation of growth and dormancy in pea axillary buds. In: VIÉMONT, J.-D.; CRABBÉ, J. (Ed.). Dormancy in plants: from whole plant behaviour to cellular control. Cambridge: University Press, 2000. p. 331-346.

21. TAMURA, F. et al. Protein changes in the flower buds of japanese pear during breaking of dormancy by chiling or hightemperature treatment. Journal of the American Society for Horticultural Science, v. 123, n. 4, p. 532-536, 1998.

Recebido em 14/05/2009 Aceito em 10/09/2009 
